\title{
CLINICAL PROFILE, FUNDUS FLUORESCEIN ANGIOGRAPHIC AND OPTICAL COHERENCE TOMOGRAPHIC FINDINGS IN CENTRAL SEROUS CHORIORETINOPATHY
}

Tariq Qureshi ${ }^{1}$ Naushin Abdulah ${ }^{2}$, Anjum Fazili ${ }^{3}$

\section{HOW TO CITE THIS ARTICLE:}

Tariq Qureshi, Naushin Abdulah, Anjum Fazili. "Clinical profile, fundus fluorescein angiographic and optical coherence tomographic findings in central serous chorioretinopathy". Journal of Evolution of Medical and Dental Sciences 2013; Vol2, Issue 34, August 26; Page: 6497-6501.

PURPOSE: To study the clinical profile, FFA and OCT findings in (CSCR). MATERIAL AND METHODS: The hospital based prospective study of 104 eyes of 100 patients was conducted in the Dept. Of Ophthalmology, GMC, Srinagar from Dec 2010 to Jan 2011.After history and complete ocular examination the clinical diagnosis of CSCR was established. FFA and OCT was done in all the 100 patients. The patterns of CSCR on FFA and OCT were observed and noted. RESULTS: In this study of 100 patients of CSCR, 95 were males and 5 were females. The range of age was between 24-45 yrs with the mean age of 34.5 yrs. 96 patients had unilateral involvement while 4 had bilateral involvement. Armed forces personnel formed a good proportion of patients. All patients presented with dimunition of vision, and scotoma with visual acuity of most of the patients in the range of $6 / 6$ to $6 / 12$. On doing FFA Ink blot pattern was seen in $88.13 \%$ of patients while smoke- stack pattern was observed in $11.86 \%$. In OCT findings neurosensory detachment was observed in 98\% and RPE abnormalities in 95\% of eyes. CONCLUSION: CSCR is usually a unilateral affliction of the eye mostly seen in young adult males presenting usually with diminution of vision or scotoma. Ink-blot pattern is more common than smoke-stack pattern on FFA. OCT could be a useful tool in clinical evaluation during the disease's acute period and resolution.

KEY WORDS: CSCR:-Central serous chorioretinopathy; FFA:- Fundus fluorescein Angiography; OCT:Optical coherence Tomography; RPE:- Retinal pigment epithelium; PED:-Pigment epithelial detachment.

INTRODUCTION: Central serous chorioretinopathy (CSCR) is a condition of unknown origin characterized by a serous detachment of the macula. It afflicts young healthy adults mostly men between the age of 20- 50 yrs (1). It is postulated to occur secondary to a leak from the choriocapillaries through the retinal pigment epithelium (RPE). (2). A recent study of choroidal perfusion suggests that choroidal ischemia might play a role in its pathogenesis. (3)The purpose of this study was to evaluate the clinical profile of CSCR and to identify the FFA \& OCT findings thereof.

MATERIAL AND METHODS: The hospital based prospective study of 100 patients (104 eyes) was conducted in the department of Ophthalmology. Govt. Medical College Srinagar from Dec 2010 to Jan 2011. The hospital is a tertiary care hospital. Criteria for inclusion of patients in the study were those having history of central visual loss with foggy vision, micropsia, metamorphopsia, and a demonstrable ring reflex on ophthalmoscopy. A complete clinical work of patient regarding age, sex, occupation, detailed history was taken. Clinical examination of the eye was made. Visual acuity testing by Snellen chart, cycloplegic refraction of the eye, slit lamp biomicroscopy using +78D fundus contact lens was done. The patient with positive fundus findings were subjective to FFA (Zeiss 


\section{ORIGINAL ARTICLE}

FF450 IR) and spectral domain OCT with resolution of $10 \mu \mathrm{m}$. FFA findings were recorded with regard to laterality, number, size \& shape of leak.

OBSERVATIONS: In the present study. 104 eyes of 100 patients were taken up for evaluation. Following data was collected from the study. Out of 100 patients, 95 were males and 5 were females, the ratio being 9:1. Maximum patients, 37 were seen in the age group of 36-40 yrs, 20 in the age group of 24-30 yrs, 28 in the age group of 31-35 yrs and no patient was seen more than 45 yrs. For age group 24-45, $x^{2}$ for $3 \mathrm{df}=11.3 . \mathrm{xc}^{2}$ (highly statistically significant) at 0.01 level of significance. Regarding the occupation, $73 \%$ were from general population, $22 \%$ were from armed forces, 5\% were from medical profession. Most of the patients were seen in the month of May and June 30 (patients) while least no of patients were recorded in December and January.Xt ${ }^{2}$ for $11 \mathrm{df}=24.7 . \mathrm{Xc}^{2}$. highly statistically significant at 0.001 level of significance. 96 patients had unilateral involvement while as 4 patients had bilateral involvement.100\% of patients presented with diminution of vision followed by scotoma in $68 \%$. Fewer patients complained of metamorphopsia (34\%), micropsia (7\%) while a negligible (4\%) of patients had complaints of either eye ache or head ache. Visual acuity of most of the patients was in the range of $6 / 6 \mathrm{p}$ to $6 / 12(50 \%)$. Very few patients had a visual acuity of less than 6/60 (about 3\%) (Table 1). Most of the eyes in our study were emetropic (89\%). Fewer eyes were hypermetropic (10\%). No eye was found to be myopic. 91\% of patients gained 1-3 lines on Snellens chart VA testing with either +0.5 Dsph or +0.75 Dsph correction.104 eyes of 100 patients with CSCR revealed a total of 118 leakage points. The leakages were either ink blots type (88.13\%) or smoke stack type $(11.86 \%)$ the former being 8 times more frequent. Most of the eyes out of 104 eyes showed a single leakage point (92 eyes) (Table 2). 4 patients out of 100 had bilateral involvement. Both eyes were involved either simultaneously or within a very short time gap. Most of the leaks occurred in superonasal quadrant followed by inferonasal, inferotemporal, superotemporal \& para foveal regions (Table3). On OCT neurosensory detachment was seen in 101 eyes (98\%), RPE abnormalities in 99 eyes (95\%). Among the RPE abnormalities. PED was seen in 70 sites (63\%) and RPE bumps in 40 sites (36\%).

DISCUSSION: The main aim of the study was to determine the clinical profile and FFA, OCT findings in patients of CSCR. As no such study was undertaken in this part before so the need for the study. Out of 100 patients, 96 had a unilateral disease at presentation. Only those patients who had a fresh leak were taken up for study. The majority of patients in the present study were males with ratio being 9:1. Youngest and oldest patients were seen in the age group of 31-40 yrs while least was seen among 41-45 yrs Armed forces personal formed a recognizable subgroup (22\%), while a very small (5\%) subgroup was formed by doctors. About one -third of our patients reported in the month of May and June while as less than (5\%) reported in the month of January and February. The commonest presenting complaint was diminution of vision in $100 \%$ of cases.VA of most of our patients (almost 50\%) was 6/12 or better. A negligible number had a VA of 6/60 or less. (Table 1). This study showed that CSCR is an acquired hypermetropic condition since a significant number of our patients gained one or two lines with a +0.50 or 0.75 DS lines. Alicia CSW et al (9) did a study on 128 patients. She observed male to female ratio of 6:1. Age group was between $26-60 \mathrm{yrs}$. 84\% of the cases were in the age group of 30-50 yrs which is almost consistent with our study. George Benette(4) made a study of 27 cases, 22 of whom conformed to the typical findings of CSCR. In his study, a 


\section{ORIGINAL ARTICLE}

significant number of patients were from naval forces which goes parallel with our record of higher incidence in armed forces. He also recorded highest number of patients in the month of January and April. But this does not coincide with our study which shows a shift towards a warmer climate. J. A Montero et al (5) made a study on 39 eyes and the mean visual acuity at baseline was 6/9 (range between 6/6 to 6/ 12) this is consistent with our study. Yameda et al (6) studied 106 eyes of 53 patients and the commonest complaint in their study was a central or paracentral scotoma (58.50\%) followed by blurred vision (34\%), metamorphopsia (18.90\%), micropsia (13.20). Our study showed scotoma to be the second commonest complaint while rest of the symptomatology is quiet parallel to that in our study. Multak and Dulton(7) made a record of 23 patients of CSR. About $70 \%$ patients were hypermetropic, $26 \%$ myopic and $4 \%$ emmetropic. This is in contrast to our conclusion where we got a majority of emmetropes and a few hypermetropes. We have not recorded myopes. FFA was done in all patients. This revealed a total of 118 leakage points which were seen as hypofluorescent spots and were divided in to smoke-stack or ink blot pattern. Ink -Blot pattern was seen in 104 out of 118 leakage points (88.13\%) while rest was smoke -stack type (11.86\%). Most of the eyes showed only a single leaking point (92 out of 104 eyes). 9 eyes showed a double leak and 3 eyes showed multiple leaks. The various distributions are shown in table 2 . In patients with bilateral CSCR ( 4 pts), 3 showed a single ink - blot leak in either eye while in the fourth eye, the R/E showed a single smoke-stack and L/E showed a single ink-blot type of leak. The quadrantic distribution of the leaking points was also characteristic. Distribution given in table 3. Shah Jamal et al (8) reported inkbolt pattern in 23 eyes (67.64\%) out of 34 eyes of 30 patients while smoke-stack appearance was seen in 11 eyes (30-35\%) which is consistent with our study. In his study unilateral involvement was seen in 26 eyes (86.66\%) while bilaterality was seen in 4 eyes (13.33\%) which goes with our study. Alicia CSW et al made a study on 128 patients of CSCR and reported 44\% of patients having more than one site of leakage not consistent with our study. Ink-bolt pattern was most common 103 patients (80\%) while smoke-stack was seen in 20 patients $(10 \%)$ which is consistent with our study. Spitznas and Huke (10) in their study of FFA pattern in 430 patients of CSR found the leakage points were mostly located in superonasal quadrant (33.2\%) followed by inferonasal quadrant (21.2\%), supero-temporal quadrant (19\%) and infero temporal quadrant (14.8\%). This is similar to our findings. Among 104 eyes, neurosensory detachment was seen in 101 eyes (98\%) and RPE abnormalities seen in 99 eyes (95\%0. Among the RPE abnormalities PED was seen in 70 sites (63\%) and RPE abnormalities or bumps were seen in 40 sites (36\%) so almost all the leaking sites were identified on OCT so it could be safer and less time consuming tool than FFA. J. A Montero (5) studied the characteristics of OCT in CSCR in 39 eyes. Neurosensory detachment was observed in 36 eyes (92\%) and RPE abnormalities in 35 eyes (89\%). R. Unnikrishnan Nair (11) studied 100 patients of acute CSCR and found PED in $70 \%$ of cases and irregular RPE distribution in 21\%.

CONCLUSION: CSCR is usually a unilateral affliction of the eye but can be bilateral in few cases. Condition mostly seen in young adult males. Presenting complaint is usually diminution of vision or a scotoma. Visual acuity is usually better than 6/12. Ink- blot leak is more common than smoke-stack pattern. Different leakage patterns are possible (ink-blot or smoke-stack) in the same patient and in same eye. Area between disc and macula was the site of predilection of leakage in majority of cases. OCT showed good efficacy to detect and measure macular changes in CSCR, being useful in clinical evaluation during the disease's acute period and resolution. 


\section{ORIGINAL ARTICLE}

Table 1:

\begin{tabular}{|l|c|c|}
\hline Visual Acuity & No of Patients & Percentage \\
\hline $6 / 6 p$ & 11 & $10.53 \%$ \\
\hline $6 / 9 p$ or $6 / 9$ & 36 & $34.66 \%$ \\
\hline $6 / 12$ & 22 & $21.15 \%$ \\
\hline $6 / 18$ & 9 & $8.65 \%$ \\
\hline $6 / 24$ & 9 & $8.65 \%$ \\
\hline $6 / 36$ & 13 & $12.5 \%$ \\
\hline $6 / 60$ and less & 4 & $3.84 \%$ \\
\hline
\end{tabular}

Table 2:

\begin{tabular}{|c|c|l|}
\hline No. of leakage points & No. of Eyes & \multicolumn{1}{|c|}{ Pattern } \\
\hline \multirow{2}{*}{ Single leak } & \multirow{2}{*}{92} & Ink blot : 82 \\
\cline { 3 - 3 } & & Smoke stack: 92 \\
\hline \multirow{2}{*}{ Double leak } & \multirow{2}{*}{9} & 1 double smoke stack \\
\cline { 3 - 3 } & & 1 smoke stack with ink blot \\
\cline { 3 - 3 } More than 2 leaks & \multirow{2}{*}{3} & 1 double ink blot \\
\hline \multirow{2}{*}{ 3 leaks ink blot } \\
\cline { 3 - 3 } & & 3 leaks ink blot \\
\cline { 3 - 3 } & & 5 leaks ink blot \\
\hline
\end{tabular}

Table 3:

\begin{tabular}{|l|c|c|}
\hline \multicolumn{1}{|c|}{ Quadrant } & No of leaks & Percentage \\
\hline Superonasal & 41 & $34.74 \%$ \\
\hline Inferonasal & 27 & $22.88 \%$ \\
\hline Inferotemporal & 24 & $20.33 \%$ \\
\hline Supertemporal & 18 & $15.25 \%$ \\
\hline Para foveal & 8 & $6.77 \%$ \\
\hline
\end{tabular}

\section{REFERENCES:}

1. Gass JD. Pathogenesis of disciform detachment of the neuroepithelium, II idiopathic central serous choroidopathy. Am J ophthalmol 1967;63:587-615

2. Spaide RF, Hall L, Hass A, Campaes L, Yamuzzi LA, Indocyanine green video angiography of older patients with central serous chorioretinopathy. Retina 1996:16.203-13

3. Kitaya N, Nagoka T, Hikichi T, Sugawera R et al. Features of abnormal choroidal circulation in central serous chorioretinopathy, Br J Ophthalmol 2003;87;709-12

4. Bennet George: Central Serous retinopathy ; BJ0 39:605-618,1977

5. JA Montero, JM Ruiz Moreno: Optical coherence tomography characterization of idiopathic central serous chorioretinopathy. Br J Ophthalmol 2005:89(5) 562-564 
6. Yamada K, Hayasaka S, Sdogewa T: Fluorence Angiographic pattern in patients with central serous chorioretinopathy at the initial visit; Ophthalmologica 1992, 205:69-76

7. Mutlak JA, Dulton GN:FFA features of acute CSR, A retrospective study; Acta Ophthalmologica, 67 (1989); 467-469

8. Shahidjamal Siddiqui, Syed Imtiyaz Ali, Mohammad Afzal, Safdar Ali Abasi, Fozia Fateh. Pattern of central serous chorioretinopathy on Fundus Fluorescein Angiography. Pak J ophthalmol 2008, Vol 24.171-175

9. Alicia CSW How, Adrain HC Koh. Angiographic characteristics of Acute Central Serous Chorioretinopathy in an Asian Population. Ann Acad Med Singapore 2006;35: 77-9

10. Spitznas m, Huke J; Number shape and topography of leakage points in acute type I CSR; Graefe's Archives Clinical EXP ophthalmol 225: 437-440, 1987

11. Dr. R. Unikrishnan Nair, S Manoj, K.G.R Nair, 69th AIOC Proceedings, Ahmedabad 2011.

\section{AUTHORS:}

1. Tariq Qureshi

2. Naushin Abdulah

3. Anjum Fazili

\section{PARTICULARS OF CONTRIBUTORS:}

1. Professor, Department of Ophthalmology, Government Medical College, Srinagar.

2. Senior Resident, Government Medical College, Srinagar.

3. Associate Professor, Department of Community Medicine, SKIMS.

\author{
NAME ADRRESS EMAIL ID OF THE \\ CORRESPONDING AUTHOR: \\ Dr. Tariq Qureshi, \\ Lal Bazar, Srinagar, \\ J \& K. \\ Email - tariqqureshi317@gmail.com
}

Date of Submission: 25/07/2013.

Date of Peer Review: 26/07/2013.

Date of Acceptance: 31/07/2013.

Date of Publishing: 23/08/2013 